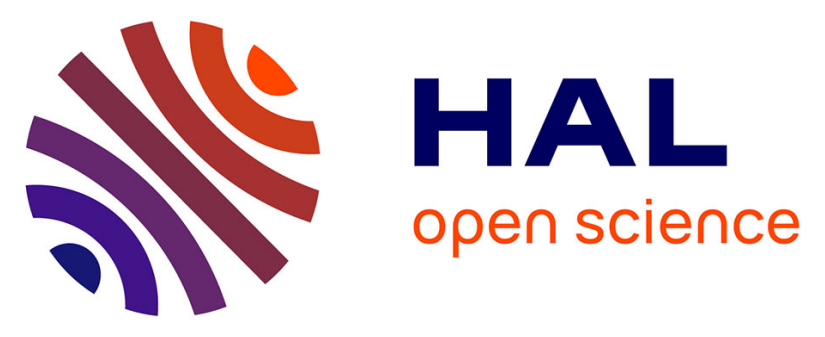

\title{
Effect of Long-Term Retention of Gadolinium on Metabolism of Deep Cerebellar Nuclei After Repeated Injections of Gadodiamide in Rats
}

Dounia El Hamrani, Véronique Vives, Rebecca Buchholz, William Même, Cécile Factor, Stefanie Fingerhut, Michael Sperling, Uwe Kärst, Philippe Robert, S. Même

\section{To cite this version:}

Dounia El Hamrani, Véronique Vives, Rebecca Buchholz, William Même, Cécile Factor, et al.. Effect of Long-Term Retention of Gadolinium on Metabolism of Deep Cerebellar Nuclei After Repeated Injections of Gadodiamide in Rats. Investigative Radiology, 2020, 55 (2), pp.120-128. 10.1097/RLI.0000000000000621 . hal-02437163

\section{HAL Id: hal-02437163 \\ https://hal.science/hal-02437163}

Submitted on 16 Nov 2020

HAL is a multi-disciplinary open access archive for the deposit and dissemination of scientific research documents, whether they are published or not. The documents may come from teaching and research institutions in France or abroad, or from public or private research centers.
L'archive ouverte pluridisciplinaire HAL, est destinée au dépôt et à la diffusion de documents scientifiques de niveau recherche, publiés ou non, émanant des établissements d'enseignement et de recherche français ou étrangers, des laboratoires publics ou privés. 


\section{Effect of long-term retention of gadolinium on metabolism of deep cerebellar nuclei after repeated injections of gadodiamide in rats}

\section{Running title: $\quad$ Gadolinium retention on cerebellar metabolism}

Dounia El Hamrani, $\mathrm{PhD}^{1}$; Véronique Vives, $\mathrm{PhD}^{2}$; Rebecca Buchholz, MSc ${ }^{3}$, William Même, $\mathrm{PhD}^{1}$; Cecile Factor, $\mathrm{PhD}^{2}$; Stefanie Fingerhut, $\mathrm{PhD}^{3}$; Michael Sperling, $\mathrm{PhD}^{3}$; Uwe Karst, $\mathrm{PhD}^{3}$; Philippe Robert, $\mathrm{PhD}^{2}$, Sandra Même, $\mathrm{PhD}^{1}$

${ }^{1}$ Center of Molecular Biophysics, CNRS UPR4301, Rue Charles Sadron, CS 80054, 45071, Orléans Cedex 2, France

${ }^{2}$ Department of Research and Innovation, Imaging and Biological Research Division, Guerbet Group, BP57400, 95943 Roissy CDG, France

${ }^{3}$ Institute of Inorganic and Analytical Chemistry, University of Münster, Corrensstraße 30, 48149, Münster, Germany

Correspondence to: Sandra Même, Center of Molecular Biophysics, CNRS UPR4301, Rue Charles Sadron, CS 80054, 45071, Orléans Cedex 2, France

Phone: +33 (0)2 382551 07; Fax: +33 (0)2 386315 17; Email: $\underline{\text { sandra.meme@ cnrs.fr }}$

\section{Acknowledgments}

The authors gratefully acknowledge: J. Leiten and M. Rasschaert (Guerbet Group, France) for their technical support in animal care; J. Valette, $\mathrm{PhD}$ (MIRCen, Gif-sur-Yvette, France) and H. Ratiney, PhD (CREATIS-INSA, Lyon, France) for their helpful discussions on MRS analysis; Y. Le Fur, PhD (CRMBM, Marseille, France) for his tutorial of CSIapo software; F. Baudimont (Atlantic Bone Screen, Nantes, France) for her expertise in histology.

\section{Conflicts of Interest and Source of Funding}

This work was supported by Region Centre-Val de Loire (France) (Grant number: n²01400093609). P.R., V.V. and C.F. are Guerbet employees. D.E., W.M., S.M., R.B., S.F., M.S., U.K. declare that there are no conflicts of interest related to this manuscript. 
Objectives: To determine potential metabolism and histological modifications due to gadolinium retention within deep cerebellar nuclei (DCN) after linear gadolinium based contrast agent injection (gadodiamide) in rats at 1 year after the last injection.

Materials and Methods: Twenty rats received 20 doses of gadodiamide $(0.6 \mathrm{mmol}$ of gadolinium per kilogram each) over 5 weeks. They were followed at 1 week (M0), 6 weeks (M1) and 54-55 weeks (M13) post-injections to evaluate hypersignal on unenhanced T1weighted MRI and metabolic alterations by ${ }^{1} \mathrm{H}$ MRS. At 1 year post-injections, brains were sampled to determine the localization of gadolinium within cerebellum by laser ablation inductively coupled mass spectroscopy (LA-ICP-MS) and to evaluate morphological changes by semi-quantitative immunofluorescence analysis.

Results: There is a significant increase of the ratio DCN/brainstem for the gadodiamide group at $\mathrm{M} 0(+7.2 \%$ vs control group $=0.989 \pm 0.01), \mathrm{M} 1(+7.6 \% v s$ control group $=1.002 \pm 0.018)$ and it lasted up to M13 ( $+4.7 \%$ vs control group $=0.9862 \pm 0.008)$. No variation among metabolic markers (cellular homeostasis, excitatory neurotransmitter and metabolites specific to a cellular compartment) were detected by ${ }^{1} \mathrm{H}$ MRS between gadodiamide and saline groups at M0, M1 and M13. At M13, LA-ICP-MS demonstrated that long-term gadolinium retention occurred preferentially in DCN. No histological abnormalities (including analysis of astrocytes, neurons and microglial cells) were found in the rostral part of DCN.

Conclusion: Repeated administration of gadodiamide lead to a retention of gadolinium preferentially within DCN until 1-year post-injections. This retention did not lead to any detectable changes of metabolic biomarkers nor histological alterations.

Key words: gadolinium based contrast agent; cerebellum; deep cerebellar nuclei; gadolinium retention; MRI ; ${ }^{1} \mathrm{H}$ MRS ; LA-ICP-MS ; histology. 


\section{Introduction}

Gadolinium is a lanthanide metal with paramagnetic properties extensively used as a contrast agent for magnetic resonance imaging (MRI). For the last 30 years, over 450 million patients have received gadolinium based contrast agent (GBCA) and these have been prescribed in 30$45 \%$ of clinical protocols. ${ }^{1-3}$ However, non-chelated gadolinium $\left(\mathrm{Gd}^{3+}\right.$ form $)$ is highly toxic in biological systems given its similar size as calcium ion $\left(\mathrm{Ca}^{2+}\right){ }^{4}$ To avoid this toxicity, $\mathrm{Gd}^{3+}$ is chelated with polyaminocarboxylate ligands which can be either macrocyclic or linear. In terms of thermodynamic and kinetic stability, it has been shown that macrocyclic chelates are more stable than their linear counterparts as they demonstrate a lower potential of gadolinium release. $^{5}$

GBCA are extracellular agents which are rapidly cleared from the intravascular space through kidney excretion in patients with normal renal function (90\% eliminated during the first 12 hours). ${ }^{6}$ Consequently, GBCA are not supposed to distribute into the central nervous system (CNS) extracellular fluid except if the blood-brain barrier (BBB) is impaired (e.g. tumors, multiple sclerosis, inflammation, irradiation treatment). ${ }^{7,8}$

Nevertheless, Kanda et al. highlighted the presence of a hypersignal on unenhanced T1-weighed MR images in deep grey matter structures of the brain (deep cerebellar nuclei, DCN and globus pallidus, GP) among patients with normal renal function who had received cumulative doses of GBCA. ${ }^{9}$ Since their seminal article, a positive correlation between this hypersignal in GP and DCN on unenhanced T1-weighed images and the number of repeated intravenous injections of GBCA (up to 6) for MRI exams has been clearly established..$^{7-11}$

Moreover, it has been extensively documented that repeated injections of linear GBCAs lead to gadolinium deposition in DCN as opposed to macrocyclic GBCAs. ${ }^{10,12-15}$ Most of the studies were conducted by inductively coupled plasma mass spectrometry (ICP-MS) on post-mortem 
samples or resections pieces. They have shown the presence of non-negligible quantities of gadolinium in brain tissue of patients. ${ }^{16,17}$ Several histological studies have demonstrated that gadolinium retention occurred without alterations of the BBB. ${ }^{16,17}$

Similar findings were reported in non-clinical rodent models assessed by MRI, ICP-MS and histology. ${ }^{18}$ Recently, Robert et al. have demonstrated that gadolinium presence persists at longterm post-injections in brain rats. ${ }^{19}$ One year after the last injection of one linear GBCA (gadodiamide), gadolinium has been detected by ICP-MS in cerebellum $(2.45 \mathrm{nmol} / \mathrm{g})$, cortical brain $(1.23 \mathrm{nmol} / \mathrm{g})$, subcortical brain $(1.52 \mathrm{nmol} / \mathrm{g})$ and brainstem $(0.74 \mathrm{nmol} / \mathrm{g})$. In the case of macrocyclic GBCA (gadoterate), gadolinium concentrations were in the range of the background noise in these structures (from 0.05 to $0.09 \mathrm{nmol} / \mathrm{g}$ ). Moreover, gadolinium detected after gadodiamide was found to be no longer in its original form but bound to macromolecules.

Several questions are still remaining, including if long-term gadolinium deposition has potential adverse health effects. In our study, we decided to address this question by following rats throughout one year after they received cumulative doses of a linear GBCA (gadodiamide). Thereby, we investigated until one year post-injections if : (i) deposition of GBCA could induce cellular alterations detectable by ${ }^{1} \mathrm{H}$ magnetic resonance spectroscopy $\left({ }^{1} \mathrm{H} \mathrm{MRS}\right)$; (ii) long-term hypersignal on unenhanced T1-weighted MR images is correlated to gadolinium deposition within DCN by laser ablation ICP-MS (LA-ICP-MS) and (iii) histological integrity of cerebellar tissue is compromised. 


\section{Materials and Methods}

\section{Animals and contrast agent injection}

All animal experiments were conducted in accordance with French regulations and in compliance with the European Union Directives 2010/63/EU. Twenty female Sprague-Dawley rats aged 10 weeks (SPF/OFA female rats, Charles River, L'Arbresle, France) were used in this study. The animals were housed under a $12 \mathrm{~h}$ light/dark cycle at constant temperature of $23 \pm 1^{\circ} \mathrm{C}$ with free access to food and water.

Two experimental groups (10 rats per group) were compared: one group received gadodiamide intravenous injections and the other control group received $0.9 \%$ saline intravenous injections. All injections were performed following a defined published protocol under general anesthesia (3\%-3.5\% isoflurane; IsoFlo, Axience, Pantin, France). ${ }^{20}$ The gadodiamide (Omniscan ${ }^{\circledR}, 0.5$ M, GE Healthcare, Chalfont-St-Giles, United Kingdom) group received 20 intravenous injections of $0.6 \mathrm{mmol}$ of gadolinium per kilogram $(1.2 \mathrm{~mL} / \mathrm{kg})$ over a period of 5 weeks (4 daily and consecutive injections per week). The daily dose of $0.6 \mathrm{mmol}$ gadolinium per kilogram is equivalent to the usual human dose of GBCAs $(0.1 \mathrm{mmol}$ of gadolinium per kilogram or $0.2 \mathrm{~mL} / \mathrm{kg}$ ), after adjustment for body surface area as recommended by the Food and Drug Administration ${ }^{21}$. A clinical follow up of animals was regularly performed.

\section{MR experiments}

During the MR experiments, the animals were positioned in a custom-built cradle to immobilize the head. They were anesthetized during the MR experiment with $1.5 \%-2 \%$ isoflurane and a mixture of $\mathrm{O}_{2} / \mathrm{N}_{2} \mathrm{O}(1: 1)$ with an output of $0.5 \mathrm{~L} / \mathrm{min}$. Respiration was monitored during the whole experiment using an air pillow and body temperature was maintained constant at $37^{\circ} \mathrm{C}$ by a warm-water circulation system. MR acquisitions were performed on a 7T horizontal ultrashielded superconducting magnet dedicated to small animal imaging (70/16 Bruker 
Pharmascan, Wissembourg, France) and equipped with a $230 \mathrm{mT} / \mathrm{m}$ gradient set. A Bruker

$11938 \mathrm{~mm}$ inner diameter birdcage coil was used for both ${ }^{1} \mathrm{H}$ transmission and reception.

120 All rats underwent the same protocol (figure 1). MR experiments were performed at M0 (one

121

122

123 week after the last injection; 0 month of washout period), M1 (six weeks after the last injection; 1 month of washout period) and M13 (54-55 weeks after the last injection; 13 months of washout period).

An unenhanced T1-weighted gradient echo sequence (intra-gate fast low angle shot; IgFLASH) was used with the following parameters: bandwidth $=75 \mathrm{kHz}$; TR/TE $=129 \mathrm{~ms} / 2.3$ ms; 6 averages; in plane resolution $=78 \times 78 \mu \mathrm{m}^{2} /$ pixel; slice thickness $=570 \mu \mathrm{m} ; 15$ slices; respiration frequency for reconstruction $=40$ breaths per minute; acquisition time $=6$ minutes 38 seconds.

PRESS (point resolved spectroscopy) sequence was acquired with the following parameters: voxel size $=3 \times 2 \times 1.5 \mathrm{~mm}^{3}$; TR/TE=2775 ms $/ 16 \mathrm{~ms}$; spectral bandwidth= $4006.41 \mathrm{~Hz}(13.34$ ppm); 2048 points; 970 averages; acquisition time $=44$ min 52 seconds. T1-weighted images were used to place the voxel in the left DCN with an extended care to avoid brainstem and subcutaneous lipids. A VAPOR module (variable pulse power and optimized relaxation delays) for water suppression was achieved to assess only cerebellar metabolites. An OVS module (outer volume suppression) was used to eliminate signal outside the voxel. Localized first and second order shim terms were adjusted within the voxel.

\section{Quantification of T1-weighted MR Image}

Quantification was performed in a blinded manner. Two regions of interest (ROI) were positioned in the most visible right or left deep cerebellar nuclei (DCN), and in the brainstem (reference). Signal intensity was calculated as the ratio between the signal of the more visible of the two DCN signal and the brainstem signal (taken as a reference). 
143 Spectra were analyzed on CSIapo software developed by Yann Le Fur (CRMBM, Marseille, 144 France) and Helene Ratiney (CREATIS - INSA, Lyon, France) which is an interface for 145 quantification algorithm QUEST (quantitation based on quantum estimation). ${ }^{22}$ QUEST is a 146 linear combination model decomposing free induction decay (FID) signal in temporal domain.

147 QUEST uses a database to fit a weighted combination of metabolite signals and then to obtain 148 a quantitation in arbitrary unit (A.U). This database is simulated through GAVA (GAmma Visual Analysis) which is used for modelling metabolites signals including their characteristics (chemical shift, amplitude and phase) and also experimental parameters (magnetic field and echo time). ${ }^{23}$

Cerebral metabolites quantified in DCN are the following: creatine and phosphocreatine $(\mathrm{Cr}+\mathrm{PCr}) ; \mathrm{N}-$ Acetyl Aspartate (NAA); choline, phosphocholine and glycérophosphocholine

154 155 (tCho); glutamate (Glu); glutamate+glutamine (Glx); Taurine (Tau) and myo-Inositol (mI) (figure 2).

\section{Sample collection}

Following MR experiments, animals were sacrificed under general anesthesia and analgesia (isoflurane, 5\%). The sacrifice consisted in exsanguination and perfusion of the blood system with saline. Immediately after euthanasia of rats, the skull was opened and the brain was taken off. The cerebellum was sampled and placed in OCT medium and then immediately cooled down in isopenthane $\left(-60^{\circ} \mathrm{C}\right)$ until complete freezing. Frozen specimens were stored at $-80^{\circ} \mathrm{C}$. The cerebellum was cut at $-20^{\circ} \mathrm{C}$ in serial $7 \mu \mathrm{m}$-thick sections at the level of deep nuclei, according to the atlas of Paxinos and Watson ${ }^{24}$. The appropriate level of section were validated with cresyl violet staining. The rostral sections of the DCN were used for immunostaining protocols and the caudal portions were sampled for LA-ICP-MS experiments. 
Slides at the level of DCN were used for the immunostaining protocol ( $n=6 /$ group) in purpose to detect 3 targets of interest: GFAP (glial fibrillary acidic protein, astrocytes maker), synaptophysin (SYP; neurons) and CD68 (microglial cells). Alexa 488 was chosen as the fluorescent dye to detect the targeted molecule. The primary antibodies and corresponding secondary antibody were selected as shown in table 1. The immunostaining protocol was performed for each marker as detailed in table 2. At the end of the immunostaining, slides were cover slipped with a medium containing DAPI (4',6-diamidino-2-phenylindole) for nuclear counterstain.

After immunostaining, images of DCN slides were acquired by epifluorescence microscopy using a FITC filter cube with similar image acquisition parameters for all slides (1280 x 1024 pixels, exposure time $300 \mathrm{~ms}$, gain 1, medium contrast). Semi-quantitative analysis of GFAP, SYP and CD68 signals were performed by evaluating the signal distribution and its prevalence on a scale from 0 (no signal) to 3 (high density of signal) within the deep cerebellar nuclei area.

\section{LA-ICP-MS}

For quantitative LA-ICP-MS analysis, matrix matched standards based on gelatin were used for external calibration. Seven gadolinium standards with concentrations ranging from 0 to $50 \mu \mathrm{g} / \mathrm{g}$ were prepared by diluting Gd ICP Standard (TraceCERT®, 1000 mg/L Gd, Sigma-Aldrich, MO, USA) and spiking 10\% w/w gelatin (Grüssing GmbH, Filsum, Germany). These standards were heated up to $40^{\circ} \mathrm{C}$ and homogenized. For quantification of iron, copper and zinc, multielement standards with concentrations ranging from 0 to $100 \mu \mathrm{g} / \mathrm{g}$ per element were prepared in the same way as the Gd standards. Concentrations of the prepared standards were verified by bulk analysis with ICP-MS (iCap TQ, Thermo Fisher, Bremen, Germany) after acidic digestions. 
190 Gelatin-based standards and the samples (n=6/group) were sliced into $10 \mu \mathrm{m}$ thick sections with

191 a cryotome (CryoStar NX70, Thermo Fisher, Bremen, Germany) and mounted onto glass slides.

192 LA-ICP-MS analysis was performed with a laser ablation system (LSX G2+, Cetac 193 Technologies, Omaha, USA) equipped with $213 \mathrm{~nm} \mathrm{Nd:YAG} \mathrm{laser,} \mathrm{a} \mathrm{two-volume} \mathrm{HelEX}$ 194 ablation cell and connective tygon tubings, connected to a ICP-MS (iCap TQ, Thermo Fisher, 195 Bremen, Germany) equipped with Ni sampler and skimmer cone and a quartz injector with an 196 inner diameter of $2.5 \mathrm{~mm}$.

197 Laser energy was adjusted to allow for complete ablation of the samples. For calibration, ten 198 parallel lines and for the samples, the whole brain section was ablated linewise with a laser spot 199 size of $25 \mu \mathrm{m}$ and a scan speed of $75 \mu \mathrm{m} / \mathrm{s}$.

\section{Statistical analysis}

201 Data are given as mean \pm SEM. Statistical analyses were performed using Prism 5.1 (GraphPad, 202 La Jolla, CA, USA). Non-parametric $\mathrm{t}$ test Mann-Whitney was used on weight, MRI and ${ }^{1} \mathrm{H}$ 203 MRS data for comparison between saline and gadodiamide groups. A $p<0.05$ was considered 204 statistically significant. 


\section{Results}

\section{Sanitary following of animals}

Four rats of the gadodiamide group died during the study period: 1 unexplained death (at M0), 3 ethically sacrificed (one at M0 and 2 at M1) because signs of pain induced by visible ulcerative and squamous skin lesions. One rat in the saline group died at M0 during the anesthesia.

\section{Qualitative analysis of T1-weighted MR images}

Figure 3 shows examples of typical non enhanced T1-weighted images obtained for both groups (saline and gadodiamide) 1 week (M0), 6 weeks (M1) and 54-55 weeks (M13) after the end of the injections.

Specific DCN signal enhancement (arrows) is present for all rats of the gadodiamide group. The hyperintensity was present on the images as early as the week following the end of the injections and persisted over time even 1 year after the end of the injections.

\section{Quantitative analysis of signal intensity in T1-weighed images}

The ratio between the signals of the DCN and the brainstem for M0, M1 and M13 are presented on figure 4. There is a significant increase of the ratio DCN/brainstem for the gadodiamide group at M0 (1 week after the end of the injections) and M1 (6 weeks after the end of the injections). This increase in DCN signal intensity lasted up to 1 year after the end of the injections.

At M0, the ratio for DCN signal intensity to brainstem increased by $7.2 \%$ in gadodiamide group (1.060 \pm 0.010$)$ compared to saline group $(0.989 \pm 0.01)$. At M1, the ratio for DCN signal intensity to brainstem was elevated of $7.6 \%$ for gadodiamide group $(1.078 \pm 0.014) v s$ saline group (1.002 \pm 0.018$)$. At M13, the ratio for DCN signal intensity to brainstem was greater of $4.7 \%$ for gadodiamide group $(1.033 \pm 0.011)$ in comparison of saline group $(0.9862 \pm 0.008)$. 


\section{MR Spectroscopy}

Figure 5 represents the concentration of metabolites measured in the DCN at M0 (1 week postinjections), M1 (6 weeks post-injections) and M13 (54-55 weeks post-injections). MRS allows to detect three categories of metabolites: (i) cellular homeostasis (creatine, choline, taurine); (ii) excitatory neurotransmitter (glutamate) and (iii) metabolites specific to a cellular compartment (NAA for neurons and myo-Inositol for glial cells). No variation among these markers was detected between gadodiamide and saline groups at M0, M1 and M13.

\section{LA-ICP-MS}

The element distribution for $\mathrm{Gd}, \mathrm{P}, \mathrm{Fe}, \mathrm{Cu}$ and $\mathrm{Zn}$ in rat brain observed after repeated injection of either gadodiamide (left side) or saline solution (right side) are shown in figure 6. Corresponding microscopic images of the analysed thin sections are also shown $(a, b)$ for comparison. Concentrations up to $64 \mathrm{nmol} / \mathrm{g}$, in a few cases even higher, were found in the brain of rats treated with gadodiamide. Highest concentrations were found in the region of DCN, with average concentrations ranging from 25 to $35 \mathrm{nmol} / \mathrm{g}$ in the three substructures. For the rat brain injected with saline solution, no Gd could be detected.

No clear correlation between gadolinium and phosphorous, copper or zinc can be observed, but the highest Gd concentrations are found in the region with highest iron concentrations. The average iron concentrations are slightly lower $(497 \mathrm{nmol} / \mathrm{g})$ in the $\mathrm{DCN}$ of the gadodiamide sample compared to the untreated animal $(665 \mathrm{nmol} / \mathrm{g})$.

\section{Immunofluorescent analysis}

Semi-quantitative analysis of cerebellar cell populations (astrocytes, neurons and glial cells) were performed at 1-year post-injections in gadodiamide and saline groups.

GFAP is a marker for astrocyte cells (density and thickness of astrocyte processes) and allowed to determine their distribution (perineuronal and perivascular). Synaptophysin is a neuronal 
253 marker which provide a morphological information on perikaryon. CD68 is a marker for 254 microglial cells which indicate their distribution (perivascular activated microglial) and their 255 morphology (diffuse neuropil) (figure 7a).

256 No morphological changes were observed between gadodiamide and saline groups based on 257 the evaluation of immunofluorescent analysis of various cells populations in the deep cerebellar 258 nuclei area (figure 7b). 


\section{Discussion}

The main goal of our study was to evaluate the long-term retention of a linear gadolinium based contrast agent (GBCA; gadodiamide) within the cerebellum, and more specifically in the deep cerebellar nuclei (DCN) after 1-year post-injections. We used both in vivo MR techniques (MRI and ${ }^{1} \mathrm{H}$ MRS) as well as quantitative chemical (LA-ICP-MS) and histological approaches.

In our experimental design, we used a rodent model receiving cumulative doses of a linear GBCA (12 mmol of gadolinium per kilogram) which was previously demonstrated to lead to a cerebellar gadolinium accumulation at 5 weeks post-injections and also at 1 year postinjections. ${ }^{19,20,25}$ Animal welfare was followed throughout our longitudinal MR study to monitor any physiological changes induced by repeated injections of gadodiamide.

This clinical monitoring allowed us to notice the apparition of skin lesions among our animals ( 3 out of 10 rats) between 1 and 5 weeks post-injections. Lohrke et al. have reported similar observations of skin injuries among 4 out of 10 rats exposed to cumulative doses of gadodiamide (50 mmol of gadolinium per kilogram; 4 times higher than in our protocol). ${ }^{26}$ In their protocol, a high concentration of gadolinium was quantified in the skin after gadodiamide exposure (1472 nmol/g) and not in the case of others GBCAs (gadopentetate dimeglumine, gadobutrol and gadoteridol). Moreover, several studies have shown that there is an accumulation of gadolinium in tissues such as bone and skin both in humans and rats with normal renal function. ${ }^{27-29}$

Throughout our longitudinal study, we observed a T1 hypersignal in cerebellum on unenhanced MR images at 1 and 5-weeks post-injections which was persistent until 13 months postinjections. This observation is in agreement with a previous report on the same rat model at 1year post-injections, gadolinium concentration was quantified up to $2.45 \mathrm{nmol} / \mathrm{g}$ within cerebellum. ${ }^{19}$ Our results obtained by LA-ICP-MS demonstrated that long-term gadolinium 
retention occurred preferentially in DCN. This result is consistent with previous studies that showed preferential gadolinium accumulation in this cerebellar structure at an earlier point $(8$ weeks post-injections). ${ }^{26}$ Given the high concentration of metals within DCN, the most likely hypothesis would be that GBCA dechelated through a transmetalation of gadolinium to iron, cupper or zinc. The limit of LA-ICP-MS method lies in its incapacity to identify whether gadolinium is present under its chelated or dechelated form.

Despite gadolinium retention within DCN, we did not observed any morphological modification of various cellular populations (neurons, astrocytes and microglia) at 1-year postinjections. Previous studies at earlier time points (8 and 20 weeks post-injections) are concordant with our results. ${ }^{26,30}$ So far, histological studies only explored morphological features. Furthers studies should focus on inflammatory markers to determine whether gadolinium retention in DCN could induce a local inflammation.

Our main goal was to identify whether repeated injections of gadodiamide could lead to metabolic alterations at short ( 1 and 6 weeks post-injections) and long-term (1-year postinjections) using ${ }^{1} \mathrm{H}$ MRS. To date, few data are available for cerebellar metabolism following repeated injections of linear GCBA. Our main observation was that long-term retention of gadodiamide did not induce any detectable metabolic changes in the cerebellum after 1-year post-injections. In accordance with literature, ${ }^{18} \mathrm{~F}$-fluorodeoxyglucose positron emission tomography $\left({ }^{18}\right.$ F-FDG-PET) imaging of rats who had received a cumulative dose of gadodiamide (13.2 mmol/kg over 7 weeks) did not reveal any abnormal glucose uptake in the DCN, and thus even with chronic cerebral hypoperfusion (model of aging and neurodegenerative diseases). ${ }^{31}$ However, a ${ }^{18}$ F-FDG-PET study had shown a lower maximum standardized uptake value (SUVmax) in DCN of patients who had received repeated injections of GBCAs (3 to 6 injections without distinction between macrocyclic and linear forms) 
compared to those who had not received, which might indicate a decrease of glucose metabolism. $^{32}$

Nevertheless, it should be taken under consideration that ${ }^{1} \mathrm{H}$ MRS may not be sensitive enough to detect any subtle modifications of cerebellar metabolism. Indeed, one technical limit of ${ }^{1} \mathrm{H}$ MRS is its inability to detect molecules below $\mathrm{mM}$ range. It also appears that metabolites involved in cellular homeostasis and excitatory neurotransmitter (glutamate) which are detectable by ${ }^{1} \mathrm{H}$ MRS may not be a target of dechelated gadolinium. Another methodological limit of in vivo ${ }^{1} \mathrm{H}$ MRS is that a technique sensitive only to molecules with a high degree of rotational molecular motion. ${ }^{33}$ Moreover, it has been recently reported that gadolinium linked preferentially to macromolecules (larger to $66.5 \mathrm{kDa}$ in insoluble fraction). ${ }^{19}$ In the case of gadolinium bounded to macromolecules such as proteins or lipids and especially within the membrane bilayer, any alteration could not be detectable by ${ }^{1} \mathrm{H}$ MRS.

In our study, we studied only one representative of linear GBCAs which is neutral in terms of charge. Indeed, linear ionic GBCAs such as gadobenate dimeglumine and gadopentetate dimeglumine induce also gadolinium retention in the cerebellum of patients. ${ }^{11,15,34}$ Furthermore, linear ionic GBCA have been previously shown to decrease the area under the peak of choline in breast and cerebral tumors. ${ }^{35-37}$ The peak of choline is widely used in diagnostic as an index for cellular density of the tumor and the degree of tumor infiltration into brain tissue. ${ }^{38}$ It will 325 be highly interesting to investigate the impact of linear ionic GBCAs on ${ }^{1} \mathrm{H}$ MRS spectra in brain. In this purpose, further studies must be conducted to evaluate if they can alter choline peak signal and thereby diagnostic.

To conclude, we demonstrate that cumulative administration of a linear and nonionic GBCA

329 (gadodiamide) induces a retention of gadolinium within DCN at 1-year post-injections and thus without altering metabolic biomarkers of cellular homeostasis and an excitatory neurotransmitter (glutamate). Although, histological analysis did not show any alterations of 
332 DCN, further studies are required to elucidate the putative clinical long-term consequences of

333 gadolinium retention (e.g. inflammatory markers) as well as the mechanism underlying such a 334 retention. 
1. Hao D, Ai T, Goerner F, et al. MRI contrast agents: Basic chemistry and safety. J. Magn. Reson. Imaging. 2012;36(5):1060-1071.

2. Kanal E. Gadolinium based contrast agents (GBCA): Safety overview after 3 decades of clinical experience. Magn. Reson. Imaging. 2016;34(10):1341-1345.

3. McDonald RJ, Levine D, Weinreb J, et al. Gadolinium Retention: A Research Roadmap from the 2018 NIH/ACR/RSNA Workshop on Gadolinium Chelates. Radiology. 2018;289(2):517-534.

4. Sherry AD, Caravan P, Lenkinski RE. Primer on gadolinium chemistry. J. Magn. Reson. Imaging. 2009;30(6):1240-1248.

5. Tedeschi E, Caranci F, Giordano F, et al. Gadolinium retention in the body: what we know and what we can do. Radiol. Med. (Torino). 2017;122(8):589-600.

6. Aime S, Caravan P. Biodistribution of gadolinium-based contrast agents, including gadolinium deposition. J. Magn. Reson. Imaging. 2009;30(6):1259-1267.

7. Errante Y, Cirimele V, Mallio CA, et al. Progressive Increase of T1 Signal Intensity of the Dentate Intravenously Administered Gadodiamide in Patients With Normal Renal Function, Suggesting Dechelation: Invest. Radiol. 2014;49(10):685-690.

8. Quattrocchi CC, Mallio CA, Errante Y, et al. Gadodiamide and Dentate Nucleus T1 Hyperintensity in Patients With Meningioma Evaluated by Multiple Follow-Up Contrast-Enhanced Magnetic Resonance Examinations With No Systemic Interval Therapy: Invest. Radiol. 2015;50(7):470-472.

9. Kanda T, Ishii K, Kawaguchi H, et al. High Signal Intensity in the Dentate Nucleus and Globus Pallidus on Unenhanced T1-weighted MR Images: Relationship with Increasing Cumulative Dose of a Gadolinium-based Contrast Material. Radiology. 2014;270(3):834-841.

10. Kanda T, Osawa M, Oba H, et al. High Signal Intensity in Dentate Nucleus on Unenhanced T1weighted MR Images: Association with Linear versus Macrocyclic Gadolinium Chelate Administration. Radiology. 2015;275(3):803-809.

11. Ramalho J, Castillo M, AlObaidy M, et al. High Signal Intensity in Globus Pallidus and Dentate Nucleus on Unenhanced T1-weighted MR Images: Evaluation of Two Linear Gadolinium-based Contrast Agents. Radiology. 2015;276(3):836-844.

12. Cao Y, Huang DQ, Shih G, et al. Signal Change in the Dentate Nucleus on T1-Weighted MR Images After Multiple Administrations of Gadopentetate Dimeglumine Versus Gadobutrol. Am. J. Roentgenol. 2016;206(2):414-419.

13. Yoo R-E, Sohn C-H, Kang KM, et al. Evaluation of Gadolinium Retention After Serial Administrations of a Macrocyclic Gadolinium-Based Contrast Agent (Gadobutrol): A Single-Institution Experience With 189 Patients. Invest. Radiol. 2017:1.

14. Bae S, Lee H-J, Han K, et al. Gadolinium deposition in the brain: association with various GBCAs using a generalized additive model. Eur. Radiol. 2017;27(8):3353-3361.

15. Radbruch A, Weberling LD, Kieslich PJ, et al. Gadolinium Retention in the Dentate Nucleus and Globus Pallidus Is Dependent on the Class of Contrast Agent. Radiology. 2015;275(3):783-791. 
16. McDonald RJ, McDonald JS, Kallmes DF, et al. Intracranial Gadolinium Deposition after Contrastenhanced MR Imaging. Radiology. 2015;275(3):772-782.

17. Kanda T, Fukusato T, Matsuda M, et al. Gadolinium-based Contrast Agent Accumulates in the Brain Even in Subjects without Severe Renal Dysfunction: Evaluation of Autopsy Brain Specimens with Inductively Coupled Plasma Mass Spectroscopy. Radiology. 2015;276(1):228-232.

18. Robert P, Frenzel T, Factor C, et al. Methodological Aspects for Preclinical Evaluation of Gadolinium Presence in Brain Tissue: Critical Appraisal and Suggestions for Harmonization-A Joint Initiative. Invest. Radiol. 2018:1.

19. Robert P, Fingerhut S, Factor C, et al. One-year Retention of Gadolinium in the Brain: Comparison of Gadodiamide and Gadoterate Meglumine in a Rodent Model. Radiology. 2018:172746.

20. Robert P, Lehericy S, Grand S, et al. T1-Weighted Hypersignal in the Deep Cerebellar Nuclei After Repeated Administrations of Gadolinium-Based Contrast Agents in Healthy Rats: Difference Between Linear and Macrocyclic Agents. Invest. Radiol. 2015;50(8):473-480.

21. FDA. FDA. Guidance for Industry: Estimating the Maximum Safe Starting Dose in Initial Clinical Trials for Therapeutics in Adult Healthy Volunteers. US Department of Health and Human Services Food and Drug Administration Center for Drug Evaluation and Research (CDER). 2005. Available at: https://www.fda.gov/downloads/drugs/guidances/ucm078932.pdf. Accessed February 5, 2018.

22. Ratiney H, Sdika M, Coenradie Y, et al. Time-domain semi-parametric estimation based on a metabolite basis set. NMR Biomed. 2005;18(1):1-13.

23. Soher BJ, Young K, Bernstein A, et al. GAVA: Spectral simulation for in vivo MRS applications. J. Magn. Reson. 2007;185(2):291-299.

24. Paxinos G, Watson C. The Rat Brain in Stereotaxic Coordinates. 6th edition. London, England: Elsevier Academic Press

25. Robert P, Violas X, Grand S, et al. Linear Gadolinium-Based Contrast Agents Are Associated With Brain Gadolinium Retention in Healthy Rats: Invest. Radiol. 2016;51(2):73-82.

26. Lohrke J, Frisk A-L, Frenzel T, et al. Histology and Gadolinium Distribution in the Rodent Brain After the Administration of Cumulative High Doses of Linear and Macrocyclic Gadolinium-Based Contrast Agents: Invest. Radiol. 2017;52(6):324-333.

27. Tweedle MF, Wedeking P, Kumar K. Biodistribution of radiolabeled, formulated gadopentetate, gadoteridol, gadoterate, and gadodiamide in mice and rats. Invest. Radiol. 1995;30(6):372-380.

28. Pietsch H, Lengsfeld P, Jost G, et al. Long-term retention of gadolinium in the skin of rodents following the administration of gadolinium-based contrast agents. Eur. Radiol. 2009;19(6):1417-1424.

29. White GW, Gibby WA, Tweedle MF. Comparison of Gd(DTPA-BMA) (Omniscan) Versus Gd(HPDO3A) (ProHance) Relative to Gadolinium Retention in Human Bone Tissue by Inductively Coupled Plasma Mass Spectroscopy: Invest. Radiol. 2006;41(3):272-278.

30. Smith APL, Marino M, Roberts J, et al. Clearance of Gadolinium from the Brain with No Pathologic Effect after Repeated Administration of Gadodiamide in Healthy Rats: An Analytical and Histologic Study. Radiology. 2017;282(3):743-751.

31. Arena F, Bardini P, Blasi F, et al. Gadolinium presence, MRI hyperintensities, and glucose uptake in the hypoperfused rat brain after repeated administrations of gadodiamide. Neuroradiology. 2019;61(2):163-173. 

PET/CT Uptake in the Dentate Nucleus and the Globus Pallidus? A Pilot Study. J. Nucl. Med. Technol. 2017;45(1):30-33.

33. Williams E, Hamilton JA, Jain MK, et al. Natural Abundance Carbon-13 Nuclear Magnetic Resonance Spectra of the Canine Sciatic Nerve. Science. 1973;181(4102):869-871.

34. Schlemm L, Chien C, Bellmann-Strobl J, et al. Gadopentetate but not gadobutrol accumulates in the dentate nucleus of multiple sclerosis patients. Mult. Scler. J. 2017;23(7):963-972.

35. Lenkinski RE, Wang X, Elian M, et al. Interaction of gadolinium-based MR contrast agents with choline: Implications for MR spectroscopy (MRS) of the breast. Magn. Reson. Med. 2009;61(6):12861292.

36. Baltzer PAT, Gussew A, Dietzel M, et al. Effect of contrast agent on the results of in vivo1H MRS of breast tumors - is it clinically significant?: EFFECT OF CONTRAST AGENT ON 1H MRS OF BREAST TUMORS. NMR Biomed. 2012;25(1):67-74.

37. Murphy PS, Dzik-Jurasz ASK, Leach MO, et al. The effect of Gd-DTPA on T1-weighted choline signal in human brain tumours. Magn. Reson. Imaging. 2002;20(1):127-130.

38. Horská A, Barker PB. Imaging of Brain Tumors: MR Spectroscopy and Metabolic Imaging. 
Table 1: Primary antibodies and corresponding antibodies for immunostaining protocol

\begin{tabular}{|c|c|c|}
\hline Primary antibody & Clone & Secondary antibody \\
\hline \hline Mouse anti-CD68 IgG1 & ED1 & Anti-mouse IgG1 A488 \\
\hline Rabbit anti-SYP & YE269 & Anti-rabbit IgG A488 \\
\hline Rabbit anti-GFAP & polyclonal & Anti-rabbit IgG A488 \\
\hline
\end{tabular}

Table 2: Steps for immunostaining protocol

\begin{tabular}{|c|c|c|}
\hline Step & Reagent & Condition \\
\hline \hline Fixation of slides & Formalin $4 \%$ & 10 min at room temperature \\
\hline Rinsing & PBS1x & $3 \times 5$ min \\
\hline Serum blocking & BSA 3\% / & $1 \mathrm{~h}$ at room temperature \\
\hline Primary antibody incubation & Anti-GFAP $1 / 1000^{\mathrm{e}}$ & $\begin{array}{c}1 \mathrm{~h} \text { at room temperature } \\
1 \mathrm{~h} \text { at room temperature } \\
1 \mathrm{~h} \text { at room temperature }\end{array}$ \\
\hline Rinsing & Anti-CD68 $1 / 200^{\mathrm{e}}$ & $3 \times$ m 5 min \\
\hline Secondary antibody & PBS $1 / 600^{\mathrm{e}}$ & $1 \mathrm{~h}$ at room temperature \\
\hline Rinsing & PBS1x & $3 \times$ m 5 min \\
\hline
\end{tabular}


440

441

442

443

444

445

446

447

Figure 1: MRI, ${ }^{1} \mathrm{H}$ MRS and injection scheme. MR experiments are performed at M0 (Week 6, one week after the last injection; 0 month of washout period), M1 (Week 11, six weeks after the last injection; 1 month of washout period) and M13 (Week 59, 53 weeks after the last injection; 13 months of washout period). After MRI, animals are sacrificed and samples are used for histological and LA-ICP-MS analysis.

Figure 2: (A) Voxel position in the left dentate cerebellar nuclei (DCN) on T1-weighed images; (B) Typical MRS spectra obtained from gadodiamide rat at 1-week post-injection. The peaks assignments are as follows: macromolecules (MM); creatine and phosphocreatine $(\mathrm{Cr}+\mathrm{PCr}) ; \mathrm{N}$-Acetyl Aspartate (NAA); choline, phosphocholine and glycerophosphocholine (tCho); glutamate (Glu); glutamate+glutamine (Glx); taurine (Tau); myo-Inositol (mI).

\section{Figure 3: T1-weighted Ig-FLASH images at 7T of saline and gadodiamide rats at M0, M1} and M13. MR images of Rat $n^{\circ} 10$ (saline group) and Rat $n^{\circ} 18$ (gadodiamide group) are qualitatively followed at the three time points.

Figure 4: Delay Effect - Quantitative analysis of enhancement at M0, M1 and M13.

Signals ratio DCN/Brainstem were measured for saline and gadodiamide groups (values are mean \pm SEM). $* * * p<0.001 ; * * p<0.01$ and $* \mathrm{p}<0.05$

Figure 5: MR Spectroscopy quantitation obtained with QUEST algorithm at delay M0, M1 and M13. Measured metabolites concentration are as follows: N-Acetyl Aspartate (NAA); creatine and phosphocreatine $(\mathrm{Cr}+\mathrm{PCr})$; choline, phosphocholine and glycerophosphocholine (tCho); taurine (Tau); myo-Inositol (mI) and glutamate (Glu). Values are mean \pm SEM. 

and saline solution (right). Phase contrast microscopic images $(a, b)$ of the analyzed thin 463 sections with quantified distribution of $\mathrm{Gd}(\mathrm{c}, \mathrm{d}), \mathrm{Fe}(\mathrm{g}, \mathrm{h}), \mathrm{Cu}(\mathrm{i}, \mathrm{j})$ and $\mathrm{Zn}(\mathrm{k}, \mathrm{l})$ as well as the 464 qualitative distribution of $\mathrm{P}(\mathrm{e}, \mathrm{f})$.

465 Figure 7: (a) Representative images of GFAP and CD68 immunostaining within deep 466 cerebellar nuclei of gadodiamide rat; (b) Semi-quantitative immunofluorescence analysis 467 of GFAP, synaptophysin (SYP) and CD68 signals. Scale were evaluated from 0 (no signal) 468 to 3 (high density of signal) within the deep cerebellar nuclei area. Values are mean \pm SEM. 